\title{
Observation of Two-Dimensional Localized Jones-Roberts Solitons in Bose-Einstein Condensates
}

\author{
Nadine Meyer, ${ }^{1,2}$ Harry Proud, ${ }^{1}$ Marisa Perea-Ortiz, ${ }^{1}$ Charlotte O’Neale, ${ }^{1,3}$ Mathis Baumert, ${ }^{1,4}$ \\ Michael Holynski, ${ }^{1}$ Jochen Kronjäger, ${ }^{1,5}$ Giovanni Barontini, ${ }^{1, *}$ and Kai Bongs ${ }^{1}$ \\ ${ }^{1}$ Midlands Ultracold Atom Research Centre, School of Physics and Astronomy, University of Birmingham, \\ Edgbaston, Birmingham B15 2TT, United Kingdom \\ ${ }^{2}$ ICFO-Institut de Ciencies Fotoniques, The Barcelona Institute of Science and Technology, 08860 Castelldefels, Barcelona, Spain \\ ${ }^{3}$ IOP Publishing, Temple Way, Bristol BS1 6HG, United Kingdom \\ ${ }^{4}$ Abaco Systems Limited, Tove Valley Business Park, Towcester, Northamptonshire NN12 6PF, United Kingdom \\ ${ }^{5}$ National Physics Laboratory, Hampton Road, Teddington, Middlesex TW11 OLW, United Kingdom
}

(Received 3 May 2017; published 11 October 2017)

\begin{abstract}
Jones-Roberts solitons are the only known class of stable dark solitonic solutions of the nonlinear Schrödinger equation in two and three dimensions. They feature a distinctive elongated elliptical shape that allows them to travel without change of form. By imprinting a triangular phase pattern, we experimentally generate two-dimensional Jones-Roberts solitons in a three-dimensional atomic Bose-Einstein condensate. We monitor their dynamics, observing that this kind of soliton is indeed not affected by dynamic (snaking) or thermodynamic instabilities, that instead make other classes of dark solitons unstable in dimensions higher than one. Our results confirm the prediction that Jones-Roberts solitons are stable solutions of the nonlinear Schrödinger equation and promote them for applications beyond matter wave physics, like energy and information transport in noisy and inhomogeneous environments.
\end{abstract}

DOI: 10.1103/PhysRevLett.119.150403

Waves play a key role in physics and technology, ranging from quantum mechanics to telecommunications. In linear media, waves spread both transversally and longitudinally due to dispersion, making them unsuitable for directed transport. This is at variance with nonlinear media, where solitary waves (solitons) become possible. In solitons, the broadening due to dispersion is counteracted by a nonlinear compression, leading to form-stable propagation at subsonic speed over large distances and particlelike properties. Depending on whether the soliton is a density dip or bump, it is classified as dark or bright, respectively. Both bright and dark solitons have been found in as diverse areas as water channels [1], high-speed data communication in optical fibers [2,3], energy transport along DNA in biology $[4,5]$, and tropospheric phenomena like Morning Glory cloud formation [6]. In experiments with ultracold atoms, bright solitons [7] and dark plane solitons (DPS) [8-12] have been extensively studied. However, with the notable exception of dipolar systems [13], bright solitons are prone to collapse. Furthermore, DPS in two or three dimensions always rapidly decay due to thermodynamical or dynamical (snaking) instabilities $[8,14,15]$. Indeed, so far, stable long-living dark solitons have been realized only in

Published by the American Physical Society under the terms of the Creative Commons Attribution 4.0 International license. Further distribution of this work must maintain attribution to the author(s) and the published article's title, journal citation, and DOI. one-dimensional systems [9-12]. In 1982, Jones and Roberts predicted a class of dark solitons that are stable both in two and three dimensions [16] and that, in contrast to DPS, have a finite extent in every direction. JonesRoberts solitons (JRS) feature indeed a distinctive elongated shape that allows propagation without change of form and, due to their finite size and area, they are expected to be immune to the snaking instability and to be resilient against scattering of thermal excitations [17]. Despite their outstanding properties, so far the experimental observation of JRSs has been elusive. In this Letter we report the experimental realization of two-dimensional JRSs, achieved by imprinting a specific phase structure onto an atomic Bose-Einstein condensate (BEC). We find that, as predicted by Jones and Roberts, these solitons are immune to both dynamical and thermodynamical instabilities and indeed their lifetime greatly exceeds the one of simple DPSs, created with the same technique. We characterize our JRSs in terms of their size, speed, and direction of propagation, finding good agreement with numerical simulations. In addition, we demonstrate that our JRSs fulfil the Kadomtsev-Petviashvili condition, as predicted by Jones and Roberts in their seminal work [16].

At the mean-field level, BECs are excellently described by a nonlinear Schrödinger equation called the GrossPitaevskii equation (GPE):

$$
i \hbar \frac{d}{d t} \Psi=\left(-\frac{\hbar^{2} \nabla^{2}}{2 m}+V+g|\Psi|^{2}\right) \Psi
$$


where $\Psi$ is the condensate mean-field wave function, $m$ the mass of the atoms, $V$ the trapping potential, and $g=$ $4 \pi \hbar^{2} a / m$ with $a$ the $s$-wave scattering length, that at low temperatures parametrizes the strength of the interatomic interactions. In their seminal paper, Jones and Roberts demonstrated that, in addition to the well-known 1D dark soliton, $\Psi=\sqrt{n_{0}} \tanh (x / \sqrt{2} \xi) e^{i g n_{0} t / \hbar}$, with $n_{0}$ the condensate density and $\xi=\left(8 \pi n_{0} a\right)^{-1 / 2}$ the healing length, Eq. (1) allows stable solitonic solutions also in two and three dimensions. They further demonstrated that the shape and properties of JRSs depend on their speed $v$. In two dimensions, for $v$ finite but lower than the speed of sound $c$, they take the form of finite line-shaped density minima, called rarefaction pulses. In the limit $v \rightarrow 0$ instead, they transform into spatially separated vortex-antivortex (VA) pairs that mutually propel each other [16]. In the first case, the vorticity vanishes and the phase pattern shows a dipolar structure of two phase winding points of opposite sign connected via an elongated phase step [18]. In the case of spatially separated VA pairs, the phase pattern consists, as one would expect, of two separated $2 \pi$ phase winding points of opposite charge. A similar picture holds for 3D systems, where JRSs take the form of axisymmetric solitary waves transitioning from rarefaction pulses to vortex rings, instead of VA pairs.

To realize our BEC, in the experiment we start loading in $10 \mathrm{~s} 10^{8}{ }^{87} \mathrm{Rb}$ atoms in a 3D Magneto-Optical Trap (MOT) fed by a 2D MOT. After an optical molasses stage the atoms are pumped in the $\left|F=2, m_{F}=2\right\rangle$ state and then magnetically transported to the science chamber by moving the trapping coils. In the science chamber the atoms are further cooled by radio frequency evaporation and then transferred into an optical dipole trap. This is produced by a single astigmatic beam at $1550 \mathrm{~nm}$, focused to a waist of $11 \mu \mathrm{m}$. The distance between the two foci is approximately $1 \mathrm{~mm}$. The atoms are transferred into the region where the beam is focused vertically, providing a strong confinement in the vertical direction and much weaker confinement in the horizontal plane. A nearly pure BEC of typically $4 \times 10^{4}$ atoms in the $|F=2\rangle$ ground state hyperfine manifold is then formed with a subsequent evaporation. The final trapping frequencies are $2 \pi \times(5,30,250) \mathrm{Hz}$, leading to an oblate BEC.

To create the JRSs, we employ a phase imprinting method [19]. The phase imprinting and imaging setup is illustrated in Fig. 1(a). A near-resonant light is reflected by a digital micromirror device and then sent onto the atoms along the vertical direction using a high-resolution optical microscope objective. Each of the $1920 \times 1080$ micromirrors can be individually controlled, allowing them to imprint on the reflected beam any arbitrary intensity pattern $I(x, y)$. The phase of the atoms is therefore locally changed by inducing a dipole potential $U_{\text {dip }}(x, y) \propto I(x, y) / \Delta$, where $\Delta$ is the detuning with respect to the atomic transition. The detection light is superimposed to the

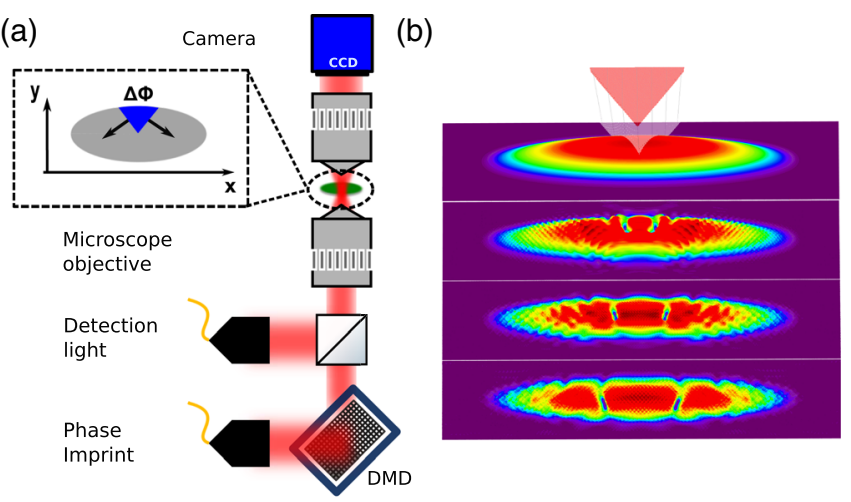

FIG. 1. (a) Experimental setup for phase imprinting. An imprint pattern displayed on a digital micromirror device (DMD) is imaged by a microscope objective onto the atoms (see inset). The detection light is superimposed on a polarizing beam splitting cube and transverses the atomic cloud, whose shadow is imaged with a second microscope objective onto the chip of a CCD camera. (b) Gross-Pitaevskii simulations of the creation of JRS via phase engineering. A triangular phase pattern whose lower vertex is approximately at the center of the BEC generates two JRSs that travel across the BEC. The time between frames is $10 \mathrm{~ms}$.

imprinting beam on a polarizing beam cube. The atoms are imaged by absorption imaging with a CCD camera using a second microscope objective that allows a resolution of $\simeq 1 \mu \mathrm{m}$.

By performing numerical simulations using the GPE, we have found that imprinting a homogeneous triangularshaped phase structure on our BEC leads to the nucleation of a couple of rarefaction pulses. After formation, each of them travels approximately in the direction perpendicular to the corresponding edge of the triangle, as shown in Fig. 1(b). The triangular shape combines two key features of JRSs: phase winding around its vertices and an elongated phase profile along its edges. To identify the most efficient way to create the JRSs, we have performed a systematic numerical study changing the shape and the position of the imprinted triangle. We have found that a triangle whose lower vertex subtends an angle of $90 \mathrm{deg}$ and that imprints a phase difference of $\pi$ is the best choice to create 2 longliving JRSs. Smaller subtended angles lead to the creation of JRSs too close to the upper border of the BEC, limiting their lifetime. Larger angles instead launch the JRSs more in the direction of the short axis, also shortening the time they can travel through the BEC. Finally, we have found that imprinting a phase step lower than $0.9 \pi$ leads to the same effect as imprinting a triangle with a smaller subtended angle. For these reasons, in the experiment the imprinted triangle has the lower vertex positioned at the center of the BEC and subtends an angle of $90 \mathrm{deg}$. We illuminate the BEC with a light pulse at approximately $10 \mathrm{GHz}$ detuning from the $|F=2\rangle \rightarrow\left|F^{\prime}=3\right\rangle$ transition for $28 \mu$ s to create a phase difference of $\pi$. Before performing absorption imaging on the BEC along the vertical direction, we then allow a time of flight of $10 \mathrm{~ms}$. 
The experimental evolution that follows the phase imprinting is displayed in Fig. 2(a). In accordance with our simulations [Fig 2(b)], we observe that the imprinting generates two elliptical rarefaction pulses, one on each side of the triangle. To characterize the motion and the properties of the two traveling JRSs, we perform two independent Gaussian fits determining their angle $\theta$, their position, their depth $n_{0}$, and their major $(\alpha)$ and minor $(\beta)$ axis lengths. We observe that, after the first $5 \mathrm{~ms}$ in which the initial sharp triangular imprint decays into the two JRSs, these latter notably travel through the BEC without any form of decay for at least $40 \mathrm{~ms}$. At this time, they stop since they reach the border of the BEC. Indeed, as reported in Fig. 3(a), we observe that until that time, their relative depth $n_{0} / n$, where $n$ is the density of the unperturbed BEC, remains approximately constant for the whole evolution. The absence of decay as well as the overall dynamical evolution are in agreement with the GPE simulations demonstrating that JRSs are stable solutions of the nonlinear Schrödinger (a)
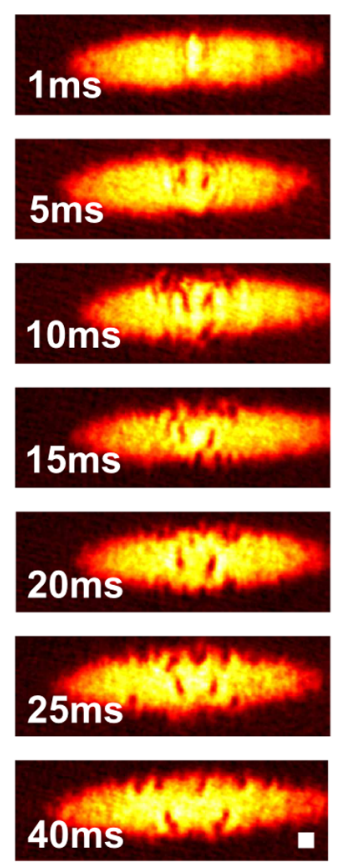

(b)
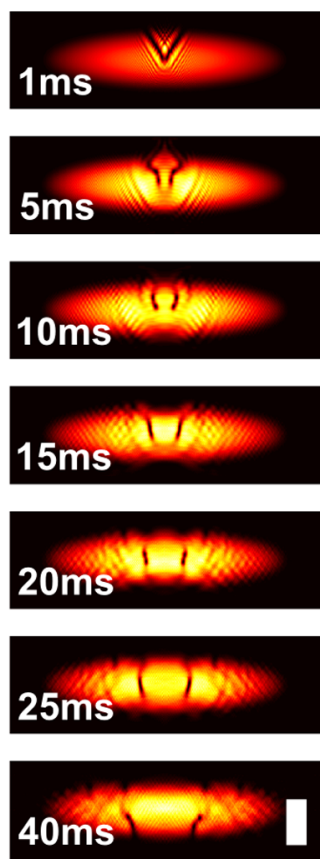

FIG. 2. (a) Experimental column density profiles of the BEC taken at different times after the initial imprinting. The time of flight is $10 \mathrm{~ms}$. (b) Corresponding numerical simulations (in trap) using the Gross-Pitaevskii equation. In both (a) and (b), two elongated but spatially localized rarefaction pulses (density dips), known as Jones-Roberts solitons, are formed close to the center of the BEC after $5 \mathrm{~ms}$ from the initial imprinting. After formation, the two rarefaction pulses maintain an almost constant finite size until they reach the border of the BEC. The discrepancies between (a) and (b) are mainly due to the switching off of the trapping potential, that is not simulated in (b). The density defects that are visible at the border of the BEC are due to unavoidable spurious sound waves stemming from the phase imprint and are unrelated to the physics of the JRS. equation. Indeed, from our results we can conclude that finite temperature effects and quantum fluctuations [20] do not significantly alter the dynamics of JRSs.

To provide a direct comparison, we also study the evolution of a standard DPS created in our experiment imprinting a linear phase step of $\pi$ on the BEC. After only $\simeq 10 \mathrm{~ms}$ we observe the DPS decaying due to snaking and thermodynamic instability into a pair of vortices, as shown in Fig. 3(b). The corresponding depth as a function of time is also reported in Fig. 3(a). The lifetime obtained by an exponential fit is $4 \mathrm{~ms}$ [21], therefore, an order of magnitude lower than the lifetime of our JRSs. This latter is limited only by the finite extension of our BEC.

Interestingly, when reaching the border of the BEC, each JRS breaks into a VA pair [18,22], as shown in the inset of Fig. 3(a). This is due to the fact that at this point the speed of the JRSs rapidly drops to zero, making the rarefaction pulses transition to separated VA pairs, as predicted by Jones and Roberts [16]. This observation, in agreement with our simulations, further confirms that our solitons belong to the Jones-Roberts class. It is worth noticing that the high trapping frequency in the vertical direction prevents the formation of vortex rings. Indeed, the smallest vortex ring has a size comparable to 4 times the healing length $\xi$. For our BEC the healing length is $\simeq 420 \mathrm{~nm}$ while the Thomas-Fermi radius in the vertical direction is $1.1 \mu \mathrm{m}$. Therefore, our condensate cannot support the formation of vortex rings but only of VA pairs along the compressed vertical direction. From this we conclude that even though our BEC is not strictly two dimensional, it can only support 2D JRSs.

By measuring the speed of propagation we confirm the subsonic nature of our JRSs, as they move with an average speed of $0.43 \mathrm{~mm} / \mathrm{s}$, which is smaller than the speed of sound of $1.21 \mathrm{~mm} / \mathrm{s}$. Interestingly, in Ref. [23] it was predicted that in two dimensions JRSs should become vortex-antivortex pairs for velocities below $\simeq 0.43 c$. Despite the fact that our JRSs travel at $0.355 c$, in our experiment they appear as pure rerefaction pulses and we attribute this discrepancy with theory to the nonuniformity of our trapped BEC. Furthermore, a single JRS is expected to travel parallel to its minor axis [16]. However, the direction of travel $\phi$ of our two JRSs features a different angle, as reported in Fig. 3(c). While $\phi$ is very close to $\pi / 4$ - the angle of the edge of the imprinting triangle- the orientation $\theta$ of the two JRSs tends to a more (anti)parallel configuration. This discrepancy could again be attributed to the nonhomogeneity of our BEC but could also stem from the fact that each of the two JRSs propagates in the velocity field exerted by the other. Given the peculiar dipolar phase structure of the JRSs, this could suggest that JRSs might feature a dipolarlike interaction. As with our imprinting method, including simulations, it is difficult to isolate one effect from the other, we plan to study this effect in detail in future work. 

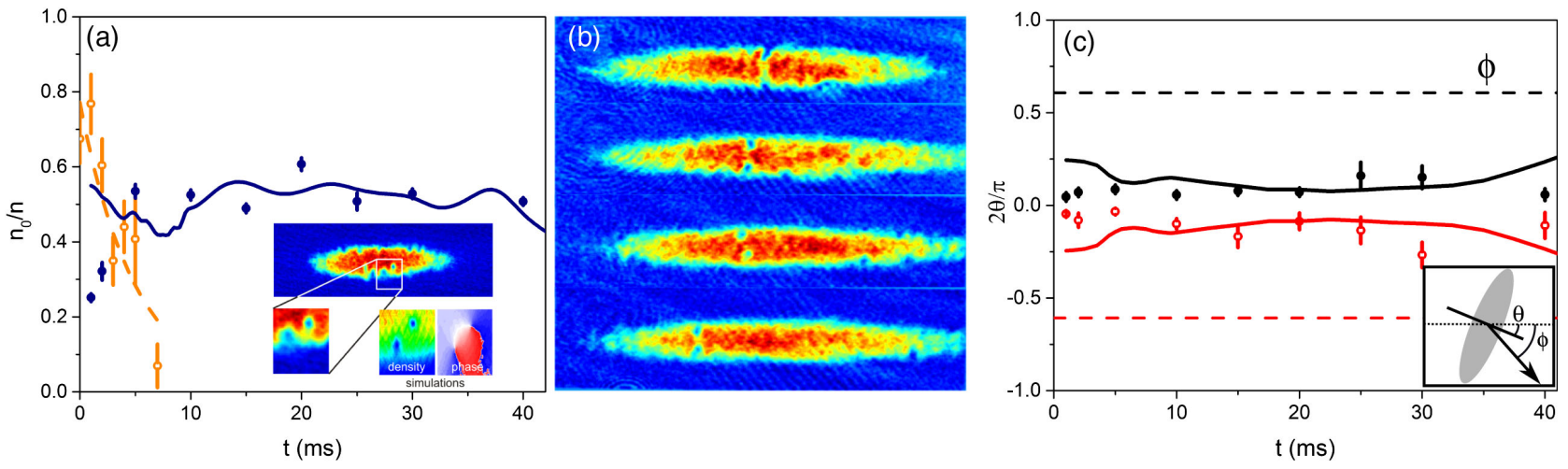

FIG. 3. (a) Points: measured relative depth over time for the JRS (filled blue) and DPS (open orange) as a function of time. Error bars are one $\sigma$ statistical error. The blue solid line is the depth predicted by the numerical simulations rescaled to take into account the finite resolution of our imaging system $(\simeq 1 \mu \mathrm{m})$. The orange dashed line is an exponential fit to the data. The inset shows an absorption image of our JRSs reaching the border of the BEC and breaking into VA pairs. The experimental observations are in good agreement with the simulations. The phase structure illustrates that the phase winds in opposite directions around the two winding points. (b) Experimental column density profiles of DPS at different evolution times showing the decay due to snaking instability. (c) Points: measured orientation of the JRSs. Error bars are one $\sigma$ statistical error. Filled black and open red points correspond to solitons traveling towards left and right, respectively. The solid lines are the prediction from the numerical simulations. The orientation shows small variations over time due to sound waves traveling through the BEC and a substantial discrepancy with respect to the direction of travel $\phi$, indicated by the dashed lines. The inset shows the definition of the angles $\theta$ and $\phi$.

By analyzing the dynamics of their size, we can gain further insights on our JRSs. As reported in Fig. 4, after an initial time of $5 \mathrm{~ms}$ in which the initial triangular structure decays into the two JRSs, their major axis $\alpha$ reaches a stable value that is kept until the solitons reach the border of the BEC. This behavior coincides with the predictions of our numerical simulations [solid line in Fig. 4(a)]. The size of the minor axis $\beta$ is at the limit of our resolution also after $10 \mathrm{~ms}$ of expansion and we do not observe any significant change, as also expected from the simulations [Fig. 4(b)]. As can be seen in Fig. 4, once they are formed, our JRSs acquire a shape that fulfils the Kadomtsev-Petviashvili condition (KPC) [24] $\alpha=C / \chi^{2}$ and $\beta=C / \chi$ (dotted lines), with $\chi=\sqrt{1-v / c} / 3$ and $C \simeq \xi / 3$. The fulfilment of the KPC is another characteristic feature of twodimensional JRSs with finite velocity [17,23]. Intuitively, an increasing axis length over time in both directions due to dissipation would be expected, similar to DPS becoming wider and faster due to thermodynamic dissipation, as observed in previous experiments [10]. Notably, as far as our experiment can test, the fulfilment of the KPC provides an outstanding immunity against both the snaking and the thermodynamic instability, making the scattering of sound waves also negligible [22].

In summary, we have experimentally realized and characterized JRSs and confirmed that they are stable solutions of the nonlinear Schrödinger equation, a longsought goal since their prediction in 1982 [16,23]. By studying their motion and shape, we have confirmed the fulfilment of the KPC and found discrepancies with respect to the original theory that might be due to the nonuniformity of our trapped $\mathrm{BEC}$ or to the interaction between the two solitons. All this creates an experimental opportunity to investigate the contribution of JRSs to the specific heat of the BEC that possibly exceeds the phonon
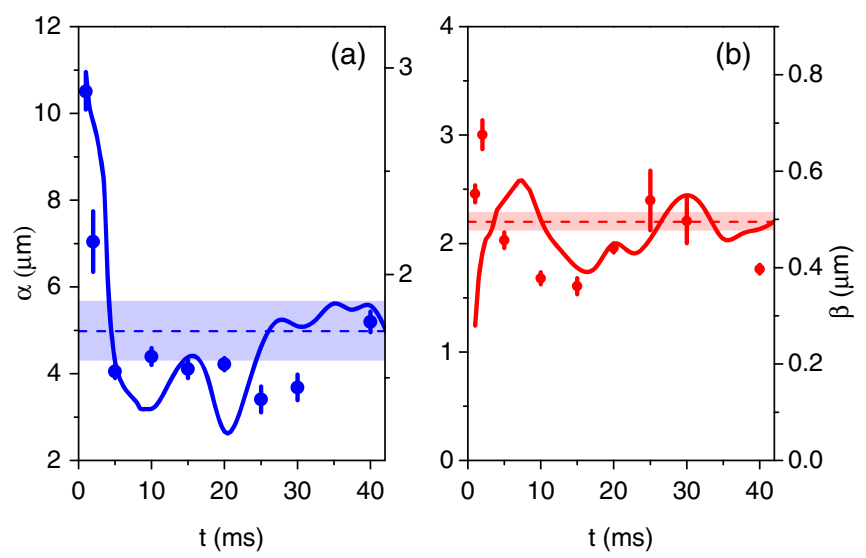

FIG. 4. (a) Evolution of the long axis $\alpha$ of the JRSs as a function of time. Points are the experimental data while the solid line is the result of the numerical simulations. The small wiggles are due to sound waves traveling across the BEC. The scale on the left (right) relates to the experimental data (simulations). The scaling factor of 3 comes from the $10 \mathrm{~ms}$ time of flight and is in accordance with the numerical simulations. The dashed line (related to the scale on the right) is the mean value of the KPC for times $>5 \mathrm{~ms}$, and the shaded band takes into account the fluctuations of the speed of the solitons. (b) The same as (a) but for the short axis $\beta$. The different scaling factor takes into account also the limited resolution of our imaging $(\simeq 1 \mu \mathrm{m})$. Error bars for (a) and (b) are one $\sigma$ statistical error. 
contribution [25]. Furthermore, studying the onset of vortex-free rarefaction pulses can also shed light on the anomalous critical scaling in acoustic turbulences described by the Kardar-Parisi-Zhang equation [26], which is relevant in turbulent systems far from equilibrium such as avalanches [27], formation of fire fronts [28], and surface growth [29]. The outstanding resilience of JRSs against dynamical instabilities and thermal decay might allow their propagation in disordered media, suggesting that they can play a significant role in many areas of science. This encourages the search for similar phenomena in other areas of physics, chemistry, and biology and opens up novel technology opportunities in directed transport through homogeneous but nonlinear media.

The data presented here are available from the research data management system of the University of Birmingham, accessible online [30].

We gratefully thank J. Brand and T. Gasenzer for discussions. This work was funded by the EPSRC (EP/ H009914/1) and the Leverhulme trust.

*g.barontini@bham.ac.uk

[1] J. Scott-Russell, Report on waves, Proc. R. Soc. Edinburgh, Sect. A 319 (1844).

[2] Y. S. Kivshar and B. Luther-Daviesb, Dark optical solitons: Physics and applications, Phys. Rep. 81, 298 (1998).

[3] Y.S. Kivshar and G. Agrawal, Optical Solitons: From Fibers to Photonic Crystals (Academic Press, New York, 2003).

[4] B. Lautrup, R. Appali, A. D. Jackson, and T. Heimburg, The stability of solitons in biomembranes and nerves, Eur. Phys. J. E 34, 57 (2011).

[5] T. Heimburg and A. D. Jackson, On soliton propagation in biomembranes and nerves, Proc. Natl. Acad. Sci. U.S.A. 102, 9790 (2005).

[6] D. R. Christie, K. J. Muirhead, and A. L. Hales, Intrusive density flows in the lower troposphere: A source of atmospheric solitons, J. Geophys. Res. 84, 4959 (1979).

[7] L. Khaykovich et al., Formation of a matter-wave bright soliton, Science 296, 1290 (2002); B. Eiermann, T. Anker, M. Albiez, M. Taglieber, P. Treutlein, K. P. Marzlin, and M. K. Oberthaler, Bright Bose-Einstein Gap Solitons of Atoms with Repulsive Interaction, Phys. Rev. Lett. 92, 230401 (2004).

[8] B. P. Anderson, P. C. Haljan, C. A. Regal, D. L. Feder, L. A. Collins, C. W. Clark, and E. A. Cornell, Watching Dark Solitons Decay into Vortex Rings in a Bose-Einstein Condensate, Phys. Rev. Lett. 86, 2926 (2001).

[9] C. Becker, S. Stellmer, P. Soltan-Panahi, S. Dörscher, M. Baumert, E.-M. Richter, J. Kronjäger, K. Bongs, and K. Sengstock, Oscillations and interactions of dark and darkbright solitons in Bose-Einstein condensates, Nat. Phys. 4, 496 (2008).

[10] S. Burger, K. Bongs, S. Dettmer, W. Ertmer, K. Sengstock, A. Sanpera, G. V. Shlyapnikov, and M. Lewenstein, Dark
Solitons in Bose-Einstein Condensates, Phys. Rev. Lett. 83, 5198 (1999).

[11] J. Denschlag et al., Generating solitons by phase engineering of a Bose-Einstein condensate, Science 287, 97 (2000).

[12] K. E. Strecker, G. B. Partridge, A. G. Truscott, and R. G. Hulet, Formation and propagation of matter-wave soliton trains, Nature (London) 417, 150 (2002).

[13] P. Pedri and L. Santos, Two-Dimensional Bright Solitons in Dipolar Bose-Einstein Condensates, Phys. Rev. Lett. 95, 200404 (2005); G. Gligorić, A. Maluckov, M. Stepić, L. Hadzievski, and B. A. Malomed, Two-dimensional discrete solitons in dipolar Bose-Einstein condensates, Phys. Rev. A 81, 013633 (2010).

[14] P. O. Fedichev, A. E. Muryshev, and G. V. Shlyapnikov, Dissipative dynamics of a kink state in a Bose-condensed gas, Phys. Rev. A 60, 3220 (1999).

[15] A. Muryshev, G. V. Shlyapnikov, W. Ertmer, K. Sengstock, and M. Lewenstein, Dynamics of Dark Solitons in Elongated Bose-Einstein Condensates, Phys. Rev. Lett. 89, 110401 (2002).

[16] C. Jones and P. H. Roberts, Motions in a Bose condensate. IV. Axisymmetric solitary waves, J. Phys. A 15, 2599 (1982).

[17] S. Tsuchiya, F. Dalfovo, and L. Pitaevskii, Solitons in twodimensional Bose-Einstein condensates, Phys. Rev. A 77, 045601 (2008).

[18] L. A. Smirnov and V.A. Mironov, Dynamics of twodimensional dark quasisolitons in a smoothly inhomogeneous Bose-Einstein condensate. Phys. Rev. A 85, 053620 (2012).

[19] L. Dobrek, M. Gajda, M. Lewenstein, K. Sengstock, G. Birkl, and W. Ertmer, Optical generation of vortices in trapped Bose-Einstein condensates, Phys. Rev. A 60, R3381 (1999).

[20] A. Syrwid, M. Brewczyk, M. Gajda, and K. Sacha, Singleshot simulations of dynamics of quantum dark solitons, Phys. Rev. A 94, 023623 (2016).

[21] Our zero-temperature GPE numerical simulations show the onset of the dynamical instability (bending and breaking of the soliton) on the same time scale of the experiment; however, we cannot reproduce the fast decay of the amplitude that we observe in the experiment. We therefore attribute this to finite temperature effects, i.e., to the thermodynamic instability. Further investigations on this effect are in order.

[22] V. A. Mironov and L. A. Smirnov, Scattering of twodimensional dark quasi-solitons by smooth inhomogeneities in a Bose-Einstein condensate, Phys. Wave Phenom. 21, 62 (2013).

[23] C. Jones, S. J. Putterman, and P. H. Roberts, Motions in a Bose condensate, V. Stability of solitary wave solutions of non-linear Schrodinger equations in two and three dimensions, J. Phys. A 19, 2991 (1986).

[24] B. B. Kadomtsev and V. I. Petviashvili, On the stability of solitary waves in weakly dispersing media, Sov. Phys. Dokl. 15, 539 (1970).

[25] S. Tsuchiya, F. Dalfovo, C. Tozzo, and L. Pitaevskii, Stability and excitations of solitons in 2D Bose-Einstein condensate, J. Low Temp. Phys. 148, 393 (2007). 
[26] S. Mathey, T. Gasenzer, and J. M. Pawlowski, Anomalous scaling at nonthermal fixed points of Burgers' and GrossPitaevskii turbulence, Phys. Rev. A 92, 023635 (2015).

[27] C. M. Aegerter, R. Günther, and R. J. Wijngaarden, Avalanche dynamics, surface roughening, and self-organized criticality: Experiments on a three-dimensional pile of rice, Phys. Rev. E 67, 051306 (2003).
[28] J. Maunuksela, M. Myllys, J. Timonen, M. J. Alalva, and T. Ala-Nissila, Kardar-Parisi-Zhang scaling in kinetic roughening of fire fronts, Physica (Amsterdam) 266A, 372 (1999).

[29] F. D. Aarão Reis, Universality in two-dimensional KardarParisi-Zhang growth, Phys. Rev. E 69, 021610 (2004).

[30] http://epapers.bham.ac.uk/3028/. 